\title{
Early predicting indicators of conversion from mild to moderate in overseas-imported COVID-19 cases
}

\author{
Shuo Liang ${ }^{1 \# \wedge}$, Zhe Yu ${ }^{2 \#}$, Yu-Yan $\mathrm{Ma}^{3}$, Chang-Feng Guo ${ }^{4}$, Hong-Zhou Lu ${ }^{5}$, Jin-Fu $\mathrm{Xu}^{1 \wedge}$ \\ ${ }^{1}$ Department of Respiratory and Critical Care Medicine, Shanghai Pulmonary Hospital, Tongji University School of Medicine, Shanghai, China; \\ ${ }^{2}$ Department of Nutrition, Shanghai Public Health Clinical Center, Shanghai, China; ${ }^{3}$ Department of Infectious Diseases, Zhongshan Hospital, \\ Shanghai, China; ${ }^{4}$ Emergency Department, Shanghai Tenth People's Hospital, Shanghai, China; ${ }^{5}$ Shanghai Public Health Clinical Center, Shanghai, \\ China \\ Contributions: (I) Conception and design: S Liang, JF Xu; (II) Administrative support: HZ Lu, JF Xu; (III) Provision of study materials or patients: \\ Z Yu, YY Ma, CF Guo; (IV) Collection and assembly of data: S Liang, Z Yu; (V) Data analysis and interpretation: S Liang, Z Yu; (VI) Manuscript \\ writing: All authors; (VII) Final approval of the manuscript: All authors. \\ \#These authors contributed equally to this work and considered as co-first authors. \\ Correspondence to: Jin-Fu Xu. Department of Respiratory and Critical Care Medicine, Shanghai Pulmonary Hospital, Tongji University School of \\ Medicine, Shanghai, China. Email: jfxucn@163.com; Hong-Zhou Lu. Shanghai Public Health Clinical Center, Shanghai, China. \\ Email: luhongzhou@fudan.edu.cn.
}

Background: Due to the ongoing pandemic of coronavirus disease 2019 (COVID-19) in foreign countries and regions, many overseas people arrive in China by air. Currently, most of the new cases of COVID-19 were imported from overseas. Here, we evaluated the predictive effect of the level of blood albumin (ALB) and serum prealbumin (PA) level in overseas-imported cases on the conversion of mild COVID-19 to moderate and its value in guiding nutritional support for these travelers.

Methods: We retrospectively analyzed serum levels of ALB and PA of 193 patients with imported COVID-19 admitted to the Shanghai Public Health Clinical Center at the time of admission on April 8, 2020.

Results: Since the first overseas-imported case was admitted to Shanghai on March 5, 2020, 195 overseasimported cases have been treated in the Shanghai Public Health Clinical Center. The disease was mild or moderate. A total of 193 patients (111 males and 82 females) entered our analysis and the disease was moderate in 108 patients and mild in 85 patients. Patients were aged 6 to 66 years (mean: 28 years). There was a strong negative correlation between the proportion of moderate type and ALB ( $\mathrm{P}=0.0073)$; thus, patients with a lower level of ALB were more likely to be diagnosed with moderate type. The correlation coefficient was close to 0 in the scatter plot, indicating that there was no linear correlation between PA and the diagnosis of moderate type $(\mathrm{P}>0.05)$. There was a strong negative correlation between age and ALB level $(\mathrm{P}<0.001)$, while length of hospital stay did not show a linear correlation with ALB or PB levels (both $\mathrm{P}>0.05)$. Therefore, older patients had lower levels of ALB and were more likely to develop moderate COVID-19.

Conclusions: The serum ALB level can be an early predictive indicator for the conversion of mild COVID-19 to moderate in cases imported overseas and may guide nutritional support.

Keywords: Serum albumin (serum ALB); serum prealbumin (serum PA); coronavirus disease 2019 (COVID-19); overseas-imported cases

Submitted Aug 26, 2021. Accepted for publication Oct 22, 2021.

doi: 10.21037/atm-21-5373

View this article at: https://dx.doi.org/10.21037/atm-21-5373

^ ORCID: Shuo Liang, 0000-0003-4748-2724; Jin-Fu Xu, 0000-0002-8039-8973. 


\section{Introduction}

Since 2020, the rapid spread of coronavirus disease 2019 (COVID-19) worldwide has posed a major challenge to global public health. On March 11, 2020, the World Health Organization (WHO) declared COVID-19 a pandemic (1). According to WHO data on April 5, 2020, the number of countries and regions affected by the pandemic of COVID-19 has reached more than 200 (2). There are no drugs for COVID-19. So early intervention is vital for these patients, as it prevents the conversion of mild disease to moderate or even severe/critical, and lowers the risk of exacerbation, making treatment more difficult. A study showed that in the early stage of COVID-19 CRP levels were positively correlated with lung lesions and could reflect disease severity (3). However, its research population is domestic patients. Due to the ongoing pandemic of COVID-19 in foreign countries and regions, many overseas people are arriving in China by air. As of April 8, Shanghai reported 213 confirmed overseas-imported cases (4). The difference between overseas-imported patients and local patients is that overseas imported patients have experienced long-distance flight travel. It has been found that the incidence of hypoproteinemia is significantly higher in cases imported overseas than in local cases, increasing the risk of developing severe disease and causing delays in recovery. So the early warning markers of overseas imported patients need to be studied. Serum albumin (ALB) and serum prealbumin (PA) level are synthesized in the liver and indicators of malnutrition. Low albumin levels on admission are associated with increased short- and long-term mortality (5). Hypoalbuminemia is associated with inflammation. Albumin acts as the main extracellular scavenger, antioxidative agent, and as supplier of amino acids for cell and matrix synthesis (6). A systematic review and meta-analysis proposed that a low albumin level can potentially lead to early recognition of severe disease (7). Here we analyzed the blood albumin (ALB) and serum prealbumin (PA) level in the recently imported cases, to learn the epidemiological characteristics of the imported cases in China, and provide evidence-based basis for nutritional support for current overseas returnees to avoid the conversion of COVID-19 from a mild type to moderate/severe types.

We present the following article in accordance with the STROBE reporting checklist (available at https://dx.doi. org/10.21037/atm-21-5373).

\section{Methods}

\section{Data sources}

Case data were obtained from the official websites of the Shanghai Municipal People's Government (http://www. shanghai.gov.cn/) and the World Health Organization (http:// www.who.int/). The study was conducted in accordance with the Declaration of Helsinki (as revised in 2013).

\section{Survey methods}

Daily data from newly confirmed cases were collected and case information from overseas-imported cases was compiled, including basic demographic data, date of onset of the disease, date of admission, date of discharge, ALB level at admission and prealbumin (PA) level at admission.

\section{Diagnostic criteria}

\section{Suspected cases}

The diagnosis of a suspected case is based on the history of contact and clinical manifestations. A diagnosis of suspicious COVID-19 was made if any epidemiological histories and two clinical manifestations were met, or if there was no clear epidemiological history, but three clinical manifestations were met.

\section{Epidemiological history}

(I) With a travel or residence history in Wuhan and its surrounding areas, or other communities with reported cases within $14 \mathrm{~d}$ before disease onset; (II) with a history of contact with patients who tested positive for SARSCoV-2 (the nucleic acid test is positive) within 14 days before disease onset; (III) with a history of contact with patients with fever or respiratory symptoms from Wuhan and surrounding areas or communities with reported COVID-19 cases within 14 daily for before disease onset; and (IV) during a cluster epidemic of COVID-19.

\section{Clinical manifestations}

(I) With fever and/or respiratory symptoms; (II) with the imaging features of COVID-19; and (III) with normal or decreased white blood cell (WBC) count and decreased lymphocyte count in the early stage.

\section{Confirmed cases}

The diagnosis of COVID-19 was confirmed if one of the following pathogenic pieces of evidence was present. 
Table 1 Baseline descriptions of mild and moderate COVID-19 cases

\begin{tabular}{lccc}
\hline Characteristics & Mild type & Moderate type & P value \\
\hline Age (years) & $21.0(17.0,29.0)$ & $31.0(22.0,42.0)$ & $<0.001$ \\
Females, $\mathrm{n}(\%)$ & $35(42.7)$ & $47(57.3)$ & 0.653 \\
BMl $\left(\mathrm{kg} / \mathrm{m}^{2}\right)$ & $24.6(22.0,25.0)$ & $24.6(23.0,25.0)$ & 0.452 \\
Albumin (g/L) & $45.7 \pm 3.3$ & $44.7 \pm 3.7$ & 0.029 \\
Prealbumin (mg/L) & $225.0 \pm 45.5$ & $229.5 \pm 60.4$ & 0.562 \\
\hline
\end{tabular}

COVID-19, coronavirus disease 2019; BMI, body mass index.

(I) Positive for SARS-CoV-2 RNA during real-time fluorescence reverse transcription PCR; (II) viral gene sequencing shows the virus is highly homologous to known SARS-CoV-2; (III) in addition to nasopharyngeal swabs, it is recommended to retain sputum as much as possible, and lower respiratory secretions can be collected for viral nucleic acid testing in patients undergoing tracheal intubation.

\section{Definition of overseas-imported cases}

Overseas-imported cases refer to patients with a history of travel or residence in a foreign country or region within 14 days before the onset of the disease, and returning to China after infection abroad.

\section{Clinical typing}

\section{Mild type}

Patients with mild clinical symptoms and no pneumonia appearance are found on imaging.

\section{Moderate type}

Patients with fever, respiratory symptoms, and/or other conditions, and pneumonia appearance is found on imaging.

\section{Statistical analysis}

Statistical analysis was performed using SPSS 22.0 software package. The potential linear correlations of ALB and PA levels with COVID-19 type, age, and duration of hospitalization were judged using the linear correlation coefficient R. Results are expressed as mean \pm standard deviations or medians (interquartiles) for continuous variables and as number (percentages) for categorical variables. Independent samples $t$-test or Mann-Whitney $\mathrm{U}$ test were used for continuous variables, and chi-square test was performed for categorical variables.

\section{Results}

Since the first overseas-imported case was admitted to Shanghai on March 5, 2020, 195 overseas-imported cases have been treated in the Shanghai Public Health Clinical Center. The disease was mild or moderate. Two cases were ruled out due to the lack of ALB and PA values. A total of 193 patients (111 males and 82 females) entered our analysis, and the disease was moderate in 108 patients and mild in 85 patients. These patients were aged 6-66 years (mean: 28 years). The mild and moderate groups showed no significant difference in terms of sex and body mass index (BMI); however, the age and ALB level significantly differed between these two groups (Table 1).

\section{Relationships of ALB level $(\mathrm{g} / \mathrm{L})$ and $P A$ level $(\mathrm{mg} / \mathrm{L})$ with the proportion of moderate type}

As shown on the scatter plot, there was a strong negative correlation between the proportion of moderate type and ALB ( $\mathrm{P}=0.0073)$; thus, patients with a lower ALB level were more likely to be diagnosed with a moderate type. The correlation coefficient was close to 0 on the scatter plot (Table 2), indicating there was no linear correlation between $\mathrm{PA}$ and the diagnosis of a moderate type $(\mathrm{P}>0.05)$ (Figure 1$)$.

\section{Relationships of ALB level $(\mathrm{g} / \mathrm{L})$ and $P A$ level $(\mathrm{mg} / \mathrm{L})$ with the age and length of hospital stay}

The scatter plot shows a strong negative correlation between age and ALB $(\mathrm{P}<0.001)$; thus, older patients tended to have lower ALB levels. However, the length of hospital stay showed no linear correlation with ALB or PB levels (both $\mathrm{P}>0.05$ ) (Figure 2). 
Table 2 Albumin levels and proportion of moderate type

\begin{tabular}{|c|c|c|c|c|}
\hline Prealbumin (PA) & Moderate type & Mild type & Total & The proportion of moderate type (\%) \\
\hline $93.64-113.64$ & 1 & & 1 & 1 \\
\hline $113.64-133.64$ & 3 & & 3 & 1 \\
\hline 133.64-153.64 & 8 & 3 & 11 & 0.727272727 \\
\hline 173.64-193.64 & 10 & 6 & 16 & 0.625 \\
\hline 193.64-213.64 & 10 & 13 & 23 & 0.434782609 \\
\hline 213.64-233.64 & 20 & 19 & 39 & 0.512820513 \\
\hline 233.64-253.64 & 13 & 16 & 29 & 0.448275862 \\
\hline 293.64-313.64 & 6 & & 6 & 1 \\
\hline 313.64-333.64 & 3 & 1 & 4 & 0.75 \\
\hline 333.64-353.64 & 1 & 1 & 2 & 0.5 \\
\hline 353.64-373.64 & 2 & 1 & 3 & 0.666666667 \\
\hline 373.64-393.64 & 1 & & 1 & 1 \\
\hline 413.64-433.64 & 1 & & 1 & 1 \\
\hline Total & 107 & 83 & 190 & \\
\hline
\end{tabular}

\section{Discussion}

China has successfully controlled the COVID-19 epidemic, and most of the new cases in this country are imported. Most imported cases are young adults (28 years in our current analysis). The symptoms were not obvious, and the disease was mild or moderate in our series. Early intervention is vital for these patients, as it prevents the conversion of mild disease to moderate or even severe/ critical, and lowers the risk of exacerbation, making treatment more difficult. According to the COVID-19 Diagnosis and Treatment Plan (trial version 7) (8) released by the National Health Commission of the People's Republic of China, it is a priority to strengthen supportive therapy and ensure adequate calories. Therefore, it is important to find effective nutritional support indicators that can reflect the changes in the disease condition, provide early warning signs of the conversion of mild COVID-19 to the moderate type, and guide the early clinical interventions.

ALB and PA levels are biochemical indicators commonly used in clinical practice to evaluate nutritional status.

Serum albumin is synthesized in the liver and has been widely recognized as a good indicator of malnutrition (9). It has been found that serum ALB concentration is associated with mortality and morbidity of nosocomial infections (10). $\mathrm{PA}$ is a protein synthesized by liver cells and is involved in the repair and binding of tissues and the transport of vitamin A and thyroxine; in addition, it is associated with a variety of respiratory diseases (11). PA is one of the negative acute-phase proteins, and its level often decreases during acute inflammation, reflecting not only the nutritional status and hepatic synthesis but also the inflammatory responses. Dynamic observation of PA level can identify whether the body is in the active phase of inflammation (12). In a recent experiment, protein deficiency resulted in malnutrition, impaired immune response, and delayed absorption of infectious lesions (13).

In our current study, there was a strong negative correlation between the proportion of moderate type and serum ALB (Table 3), suggesting patients with a lower serum 

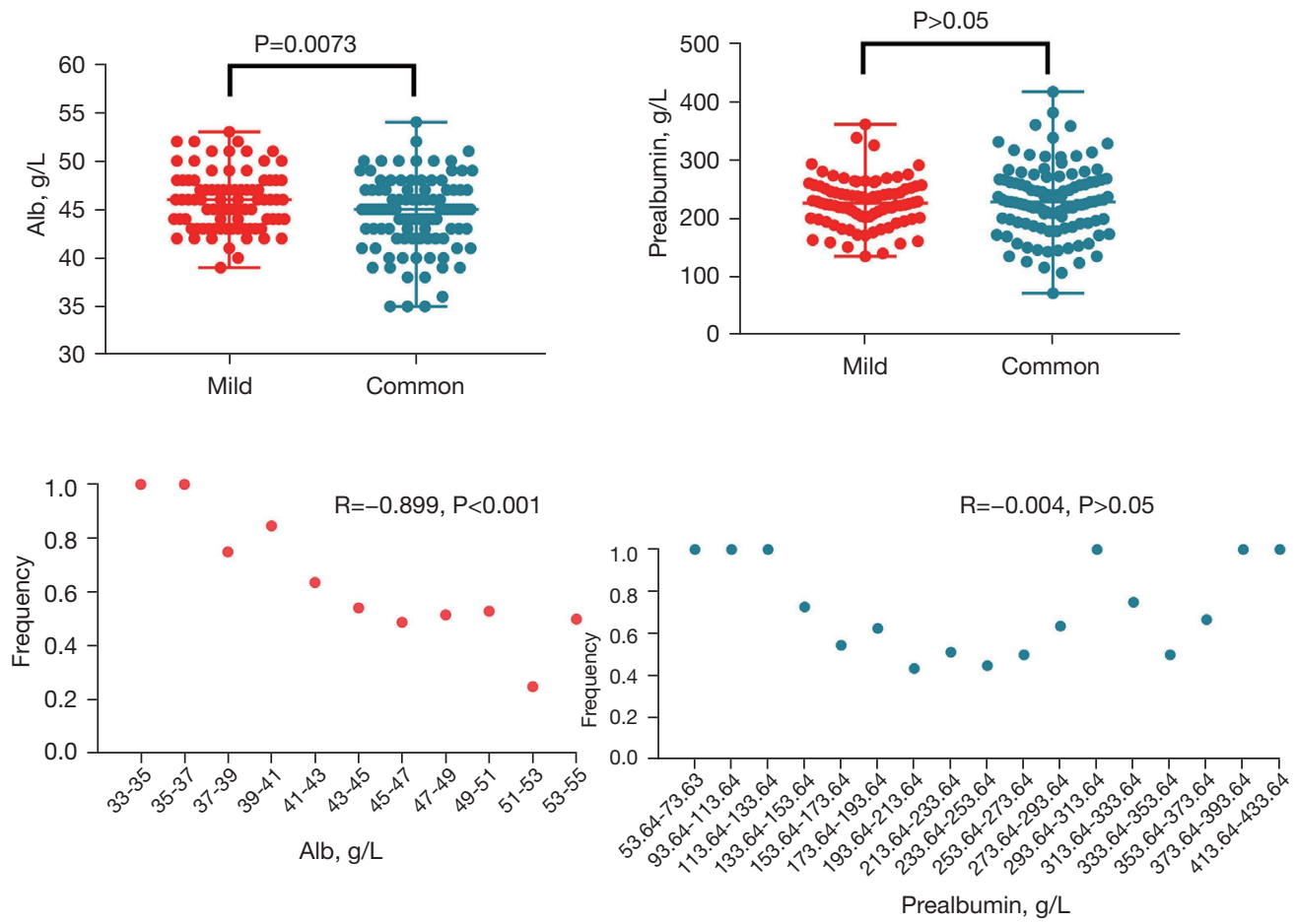

Figure 1 Relationships of ALB level $(\mathrm{g} / \mathrm{L})$ and PA level $(\mathrm{mg} / \mathrm{L})$ with the proportion of moderate type. ALB, albumin; PA, prealbumin.
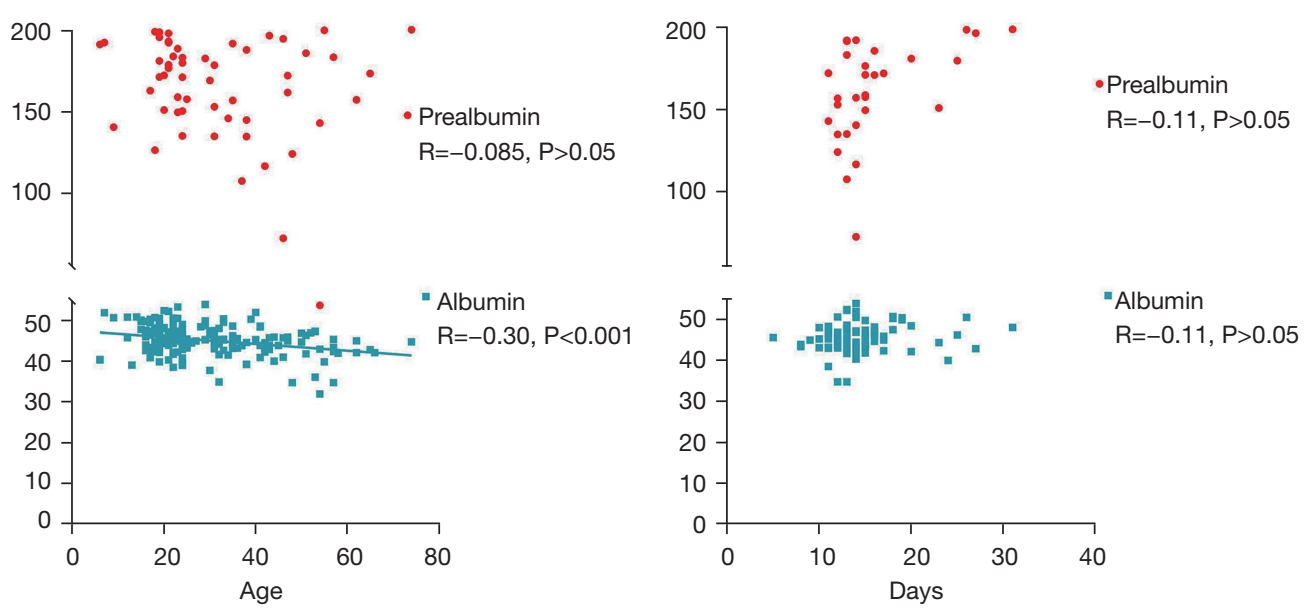

Figure 2 Relationships of ALB level (g/L) and PA level (mg/L) with the age and length of hospital stay. ALB, albumin; PA, prealbumin.

albumin ALB level are more likely to be diagnosed with moderate COVID-19. In cases where serum PA was below normal, PA was strongly negatively correlated with the confirmation of the moderate type (Table 2). Thus, patients with low PA levels are more likely to develop the moderate type. Therefore, low serum ALB and PA levels can be used as predictive indicators of malnutrition and more severe type in COVID-19 patients (Figure 1) and may be used for prognostic prediction. Both ALB and PA are at low levels in overseas-imported cases, and the risk of conversion from a mild type to moderate type is relatively high, which may be explained by the inadequate nutrition after a long airplane trip. Therefore, early provision of high-protein nutrition is especially important among international travelers. The diet should contain sufficient protein and carbohydrates during a long-haul flight, avoiding greasy and gastrointestinal 
Table 3 Albumin levels and proportion of moderate type

\begin{tabular}{lcccc}
\hline Albumin (ALB) & Moderate type & Mild type & Total & The proportion of moderate type (\%) \\
\hline $33-35$ & 3 & & 3 & 1 \\
$35-37$ & 1 & 1 & 4 & 0.75 \\
$37-39$ & 3 & 2 & 13 & 0.846153846 \\
$39-41$ & 11 & 8 & 22 & 0.636363636 \\
$41-43$ & 14 & 22 & 48 & 0.541666667 \\
$43-45$ & 26 & 23 & 45 & 0.488888889 \\
$45-47$ & 22 & 15 & 31 & 0.516129032 \\
$47-49$ & 16 & 8 & 17 & 0.529411765 \\
$49-51$ & 9 & 3 & 4 & 0.25 \\
$51-53$ & 1 & 1 & 2 & 0.5 \\
$53-55$ & 1 & 83 & 190 & 17 \\
Total & 107 & & & 1 \\
\hline
\end{tabular}

discomfort-prone foods. Patients with mild or moderate COVID-19 should receive supportive therapy to ensure adequate calories. A high-protein diet (such as eggs, poultry, and other foods) should be provided.

We also found a strong negative correlation between age and ALB level in patients with mild or moderate COVID-19, suggesting reduced ALB level is more likely to occur in older individuals (Figure 2). Thus, serum ALB monitoring is more valuable in older COVID-19 patients, and early nutritional support should be offered to avoid worsening the disease. Similarly, Liu et al. (14) first systematically reviewed the impact of age on the clinical features and important outcomes of local COVID-19 patients and concluded that the clinical characteristics and prognosis of COVID-19 differed significantly among patients in different age groups. However, since most of the overseas-imported cases in our current analysis were young adults, the results might be biased and should be further verified. A comparison of the findings between local cases and imported cases may be valuable.

Furthermore, we did not find a correlation between serum ALB/PA levels and length of hospital stay in the overseasimported patients with mild or moderate COVID-19. However, limited by the small sample size, our data had a large dispersion and needed to be further validated. Our current study also cannot determine whether the treatment of hypoproteinemia can prevent the deterioration of the patient's condition and needs further evaluation in future studies. Thankfully, the advantages of our study is the serum ALB level can be an early predictive indicator for the conversion of mild COVID-19 to moderate in cases imported overseas.

In conclusion, serum ALB and PA levels decrease in COVID-19 patients, showing a strong negative correlation with the proportion of moderate type. They may serve as an early predictive indicator for converting mild COVID-19 to a moderate type and assist clinicians in making informed decisions for patients. Timely judge whether the patient will become severe, and provide the basis for nutritional support, so as to better help the overseas-imported patients.

\section{Acknowledgments}

We thank Dr. Rong Jiang from the Department of Pulmonary Circulation, Shanghai Pulmonary Hospital, Tongji University, for her contribution to the statistical analysis and the production of icons.

Funding: This study was supported by Natural Science Foundation of China (No. 82170051), Shanghai Municipal Health Commission (No. 202140257) and Clinical Research foundation of Shanghai Pulmonary Hospital (No. FKLY20025).

\section{Footnote}

Reporting Checklist: The authors have completed the 
STROBE reporting checklist. Available at https://dx.doi. org/10.21037/atm-21-5373

Conflicts of Interest: All authors have completed the ICMJE uniform disclosure form (available at https://dx.doi. org/10.21037/atm-21-5373). The authors have no conflicts of interest to declare.

Ethical Statement: The authors are accountable for all aspects of the work in ensuring that questions related to the accuracy or integrity of any part of the work are appropriately investigated and resolved. The study was conducted in accordance with the Declaration of Helsinki (as revised in 2013).

Open Access Statement: This is an Open Access article distributed in accordance with the Creative Commons Attribution-NonCommercial-NoDerivs 4.0 International License (CC BY-NC-ND 4.0), which permits the noncommercial replication and distribution of the article with the strict proviso that no changes or edits are made and the original work is properly cited (including links to both the formal publication through the relevant DOI and the license). See: https://creativecommons.org/licenses/by-nc-nd/4.0/.

\section{References}

1. Mahase E. Covid-19: WHO declares pandemic because of "alarming levels" of spread, severity, and inaction. BMJ 2020;368:m1036.

2. World Health Organization. Available online: https:// www.who.int/

3. Shanghai Municipal People's Government. Available online: http://www.shanghai.gov.cn/

4. Wang L. C-reactive protein levels in the early stage of COVID-19. Med Mal Infect 2020;50:332-4.

Cite this article as: Liang S, Yu Z, Ma YY, Guo CF, Lu HZ, $\mathrm{Xu} \mathrm{JF}$. Early predicting indicators of conversion from mild to moderate in overseas-imported COVID-19 cases. Ann Transl Med 2021;9(20):1584. doi: 10.21037/atm-21-5373
5. Akirov A, Masri-Iraqi H, Atamna A, et al. Low Albumin Levels Are Associated with Mortality Risk in Hospitalized Patients [published correction appears in Am J Med. 2020 May;133(5):646]. Am J Med 2017;130:1465.e11-1465.e19.

6. Soeters PB, Wolfe RR, Shenkin A. Hypoalbuminemia: Pathogenesis and Clinical Significance. JPEN J Parenter Enteral Nutr 2019;43:181-93.

7. Aziz M, Fatima R, Lee-Smith W, et al. The association of low serum albumin level with severe COVID-19: a systematic review and meta-analysis. Crit Care 2020;24:255.

8. Covid-19 pneumonia diagnosis and treatment plan (trial version 7), National Health Commission of the People's Republic of China, 2020.3.8.

9. Slattery E, Patchett S. Albumin as a marker of nutrition: a common pitfall. Ann Surg 2011;254:667-8; author reply 668 .

10. Llop JM, Muñoz C, Badía MB, et al. Serum albumin as indicator of clinical evolution in patients on parenteral nutrition. Multivariate study. Clin Nutr 2001;20:77-81.

11. Schols A, Mostert R, Soeters P, et al. Inventory of nutritional status in patients with COPD. Chest 1989;96:247-9.

12. Sann L, Bienvenu F, Bienvenu J, et al. Evolution of serum prealbumin, C-reactive protein, and orosomucoid in neonates with bacterial infection. J Pediatr 1984;105:977-81.

13. Pérez H, Malavé I, Arredondo B. The effects of protein malnutrition on the course of Leishmania mexicana infection in C57Bl/6 mice: nutrition and susceptibility to leishmaniasis. Clin Exp Immunol 1979;38:453-60.

14. Liu Y, Mao B, Liang S, et al. Association between age and clinical characteristics and outcomes of COVID-19. Eur Respir J 2020;55:2001112.

(English Language Editor: J. Chapnick) 